\title{
An Effective and Scalable AODV for Wireless Ad hoc Sensor Networks
}

\author{
Srinivas Sethi \\ Dept. of CSEA \\ IGIT Sarang \\ Odisha, India
}

\author{
Ashima Rout \\ Dept. of Elect. And Etc. Engg. \\ IGIT Sarang \\ Odisha, India
}

\author{
Debajyoti Mishra \\ Dept. of Elect. And Etc. Engg. \\ IGIT Sarang \\ Odisha, India
}

\begin{abstract}
Appropriate routing protocol in data transfer is a challenging problem of network in terms of lower end-to-end delay in delivery of data packets with improving packet delivery ratio and lower overhead as well. In this paper we explain an effective and scalable AODV (called as AODV-ES) for Wireless Ad hoc Sensor Networks (WASN) by using third party reply model, n-hop local ring and time-to-live based local recovery. Our goal is to reduce time delay for delivery of the data packets, routing overhead and improve the data packet delivery ratio. The resulting algorithm "AODV-ES" is then simulated by NS-2 under Linux operating system. The performance of routing protocol is evaluated under various mobility rates and found that the proposed routing protocol is better than AODV.
\end{abstract}

\section{General Terms}

Mobile Ad hoc Network, Algorithm.

\section{Keywords}

Mobile Ad hoc Network; AODV-ES; Routing Protocol; Effective; scalable;

\section{INTRODUCTION}

With the development in wireless communication technologies, Wireless Ad hoc Sensor Network (WASN) has gained worldwide attention in recent years. A WASN consists of a large number of low cost, low power and small sensor nodes spread across a geographical area without fixed infrastructure. Each sensor node has wireless communication capability and some level of intelligence for signal processing and networking of the data. Hence, it bears great application potential in many scenarios, such as wireless sensors, industrial control and monitoring, intelligent agriculture, inventory tracking, catastrophic management, war frontier, office networks, security and taxicab networks among others. Data replication, assignment of tasks or sending of commands to a specific group of sensor nodes etc., are examples of these types of scenarios. WASN, a pair of sensor nodes communicate by sending messages either over a direct wireless link (single hop) or over a sequence of such links(multiple hop) containing one or more intermediate nodes. Direct communication between two pair of nodes is possible only when they lie within one another's transmission range. WASN has become popular in recent years because of their easy deployability less infrastructure requirement for unplanned network. It is applicable for short range radios and suitable for applications. All nodes behave as routers and take part in route discovery and maintenance of the breakage route to other sensor node(s) in the network. Without the fixed base station the sensor nodes dynamically exchange the data among themselves.

In WASN, sensor nodes are not familiar with the topology of their networks due to highly dynamic in nature. So the nodes need to discover the topology. A new node may announce its presence and should listen for announcements broadcast by its neighbors. Each sensor node learns about nearby nodes and also learns how to communicate with them. Routing protocols have vital role in these types of scenarios. Mainly, three different types of routing protocols are available and they are table driven, on-demand and hybrid routing protocols.

In Table Driven Routing Protocol, each sensor node is required to maintain a table by using periodic updates to track the changes in network topology. They periodically exchange messages among themselves. Therefore the routes to all destinations are ready to use at any movement and as a consequence initial delays before sending data are small. This approach has the advantage that a route is generally available when needed. However it requires regular routing updates which may consume a large portion of limited resources. Since queues are filled with control packets and there are more packet collisions due to more network traffic, it is also possible that the control traffic delays the data packets. DSDV is an example of this type of protocol.

On-demand type of protocol finds a route on demand, and the route is discovered on need basis. High latency time in route discovery process is the main disadvantage of this protocol. Examples of reactive routing protocol are AODV, DSR.

Hybrid Routing Protocol takes into account the advantages of both on-demand and table driven protocol. The routing is initially established with some proactively proposed routes and then serves the on-demand from additional activated nodes through reactive flooding. This is achieved mostly by proactively maintaining routes to nearby nodes and determining routes to distant nodes using an on-demand routes discovery strategy. It provides a better compromise between communication overhead and delay. It also provides better scalability. CBRP, ZRP are examples of hybrid routing protocols.

To quicken the process of route discovery and to further reduce flooding, AODV-ES adopts the third-party reply model [10] in which an intermediate node has major role between source and destination. Source and destination are treated as first and second party respectively; whereas, intermediate node can be treated as third party. Any visited intermediate nodes that have a 
route in its routing table to the same destination can generate a route reply. There is no need to forward the message to continue traveling in search for the destination. [12] The n-hop local ring of a node contains all nodes which are within n-hop away from this centre node. The radius of local ring is predefined hop count value.

Third party reply strategy in AODV-ES works efficiently with the n-hop local ring search to reduce routing overhead. Further increase the PDR and decrease the end-to-end delay of routing protocol. As a result the performance of proposed routing protocol is improved in terms of above parameters.

TTL-based Local recovery [13] is quite effective in reducing unnecessary use of bandwidth. If the broken link is closer to the destination than source, attempt a local repair. Otherwise, bring down the route.

This paper is organized as follows: section- 2 discussed the related works followed by the proposed works in section-3. In Section-4, it described the performance evaluation parameters followed by simulation parameters in section-5. Results and discussions are described in section- 6 followed by conclusions in Section-7.

\section{RELATED WORKS}

Dynamic Source Routing (DSR) [1][2] can learn more routing information from the traffic, because of DSR packets contain complete route information. As a result DSR generates fewer route discoveries. DSR is potentially able to obtain multiple routes through multiple route replies and generates more than one route by a single route discovery. In DSR, the destination replies to all valid received route requests (RREQs). While route information in DSR is maintained by the source, a distributed manner is more suitable for MANETs. In DSR, source routes carried in data packets are likely to cause significant overhead in larger networks where routes are longer. Control overhead can be reduced by creating a route maintenance mechanism between only those nodes which needs the services to transmit. Route cache can also help to cut the overhead burden. Packet header size grows with route length due to source routing.

Destination Sequenced Distance Vector (DSDV)[3] is a proactive routing protocol, based on classical Bellman ford routing algorithm for finding shortest paths between ad hoc nodes with some improvement. It is a hop-by-hop distance vector routing protocol requiring each node to periodically broadcast routing updates. The advantage of DSDV is that, it guarantees loop-freedom as compared to traditional distance vector routing protocol. No delays caused by route discovery due to all nodes are active. But it has high overhead because, most of the routing information never used in the network.

AODV [4][5] is designed from combining some feature from DSDV and DSR as well. The use of destination sequence numbers in DSDV with the on-demand route discovery technique in DSR is to formulate a loop-free, on-demand, single path and distance vector protocol. AODV is based on hop-by-hop routing approach. It is a uniform and destination based reactive routing protocol. It uses table driven routing framework and destination sequence numbers for an on demand protocol. It uses traditional routing tables with one entry per destination. It minimizes the number of required broadcasts by creating routes on demand basis. For nodes which are not selected in the path, AODV does not maintain any routing information nor takes part in the routing table exchanges. It prepares loop free routes. It disseminates information about link breakage to its neighboring nodes. The source node includes its own sequence number and broadcast id in the route request and the most recent sequence number for the destination. Intermediate nodes do reply only if they have a route to the destination, whose corresponding destination sequence number is equal to or greater than that contained in the route request.

AODV-I [6] is an extension of AODV by adding the congestion processing and the routing repair mechanism reduces the packet loss rate and the end-to-end latency. It also enhances the utilization rate of the network resources. In [7], the authors tried to reduce delay and improve packet delivery ratio by merging multipath and path accumulation with AODV routing protocol. It also reduces the routing overhead by reducing the frequency of route discovery process.

Based on the basic AODV protocols, an improved protocol is presented in [8] in which node power, load status and link state between nodes are considered route selection procedure. It has been found that the improved AODV protocol has higher package delivery ratio, lower end to end delay and lower routing overhead than basic AODV protocol.

In [11], authors have examined five different combinations of modifications with AODV in order to improve its scalability and their respective performance has been studied. They studied the use of local repair which is beneficial in increasing the number of data packets that reach their destinations. It also seems to further reduce the amount of control overhead generated by the protocol by limiting the number of nodes affected by route discoveries expanding ring search and query localization techniques.

In [16] the authors evaluate the performance of AODV routing protocol with random based entity mobility model and pursue group mobility model, in respect to PDR, Delay and Throughput. They considered for their simulation, a scenario by varying the speed of the individual nodes. They observed the pursue group mobility model performs better than random based entity mobility models.

The authors in [17] designed the protocol based on the ad hoc on-demand distance vector (AODV), introduce forwarder nodes in the sensor networks in order to extend the lifetime of the entire sensor network. The authors examined that the lifetime of the sensor network has been considerably increased by introducing the forwarder nodes.

A preemptive, self-repairing AODV (AODV-PSR) [18] scheme that is able to find an alternative link to a failing link, performs better than the ADOV-LR, due to its avoidance of packet buffering delay and excess use of control messages during link repair for MASNET. That enhances the local repair (LR) phase of the prominent ad hoc routing protocol AODV-LR and reduces the LR overhead. The authors designed the said protocol based on the early detection of a link failure and the finding in advance of an alternative link. The process also carries out the information of the alternative link without the need for the extra transmission of control messages. So AODV-PSR is a step toward providing Quality of service (QoS) over the limited power and bandwidth of MASNET. 
In this paper, we tried to control the overhead and end-to-end delay with improving the packet delivery ratio and AODV is considered for comparison with proposed AODV-ES for wireless ad hoc sensor network. The packet delivery ratio is significantly improved along with controls the overhead and end-to-end delay.

\section{PROPOSED PROTOCOL}

AODV-ES is an extension of AODV with third party reply, n-hop local ring search and time-to-live based local recovery. All the essential functionality of AODV, including route request (RREQ), route reply (RREP), route error (RRER), HELLO messages have been implemented in AODV-ES for route discovery and maintenance. RREQ, RREP messages are used for route discovery and RERR, HELLO messages are used for route maintenance. It checks the routing table to find out a route existence after AODV-ES receives a request to send a message. The router simply forwards the message to the next hop if the route exists. Otherwise, it initiates the RREQ to determine the route. Each routing table consists of the following fields

$>$ Destination address

$>$ Next hop address

$>$ Destination sequence number

$>$ Hop count

Route discovery typically involves a network-wide flood of RREQ messages targeting the destination and waiting for a RREP messages. When a node receiving a request either fresh enough route to the destination or itself the destination, the node generates a RREP message and sends this message along the reverse path backward the originating node. An intermediate sensor node receiving a RREQ packet first sets up a reverse path to the source using the previous hop of the RREQ as the next hop on the reverse path. The intermediate sensor node generates a RREP, if a valid route to the destination is available, otherwise the RREQ rebroadcasts. A forward path to the destination is established as the RREP proceeds towards the source. The RREQ originator will update its routing table with the most recent routing information; that is, it uses with the route with greatest destination sequence number. During the route discovery process if any visited intermediate sensor node that have a route in its routing table to the same destination, it can generate a route reply. This is the basic principle of third party reply. There is no need for the forward the message to continue traveling in search for the destination to control the overhead in the network. All the above can be carried out within the n-hop local ring.

Further we are trying to control the delay and overhead by using time to live (TTL) [13] of proposed routing protocol. If predefined TTL is expiring, then "stop instruction" will be broadcasted to stop the process. So that, it controls the wastage of time for the sensor node which is presently not available or switched off in the network.

Each sensor nodes in the network keeps the track of a precursor list and an outgoing list; where, a precursor list is a set of sensor nodes that route through the given node and the outgoing list is the set of next-hops that this node route through. Each sensor node periodically sends HELLO messages to its precursor, if no message has been sent to that precursor recently and each sensor node expects to receive the messages periodically from each of its outgoing sensor nodes. The node is presumed to be not reachable if no message has been received by other sensor nodes for an extended period of time. Route maintenance is done using route error (RERR) packets. When a link failure is detected, a RERR is sent back via separately maintained predecessor links to all sources using that failed link. It initiates a new route discovery if the route is still needed.

Each sensor node maintains a monotonically increasing sequence number for itself and it maintains the highest known sequence numbers for each destination in the routing table. Since destination sequence numbers are tagged on all routing messages, it provides a mechanism to determine the relative freshness of two pieces of routing information.

The AODV-ES protocol maintains an invariant that destination sequence numbers monotonically increase along a valid route, which is used to prevent routing loops. A node can receive a routing update via a RREQ or RREP packet either forming or updating a reverse or forward path. We refer to such routing updates received via a RREQ or RREP as "route advertisements."

The following procedures are given below for route discovery with Third Party Reply in local ring search and TTL-based Local Recovery of AODV-ES.

\section{Procedure: Third Party Reply in local ring search}

Begin

If(Hop count difference $<=$ local ring radius)//n-hop Local Ring Search

Begin

If (node is destination)

Send route reply message

Else if (The node is not destination)

Begin

Check route table

If(got route to destination) // Third Party Reply

Send route reply message

Else

Forward the route request message

End

End

Else

Discard Forward Query Message

End 


\section{Procedure: Forwarding Query Message}

Begin

If(received time of Forwarding Query Message $=1$ and Hop count difference $<=$ local ring radius) // to make non duplicate

Begin

Update the Forwarding Query Message

Update the Forwarding table

Broadcast the Forwarding Query Message

End

Else

Discard the Forward Query Message

End

\section{Procedure: TTL based Local Recovery}

Begin

If (node does not receive RREP within a predefined TTL value)

Begin

Broadcast the "stop instruction"

Stop

End

Else

$/ *$ if the broken link is closer to the destination than source, attempt a local repair. Otherwise, bring down the route.*/

If (number of forwards > routing hops)

Begin

// retrieve all the packets in the ifq using this link.

// queue the packets for which local repair is done.

Execute local routing repair;//local repair

Stop

End

End

In this paper the important performance evaluation parameters like End-to-End delay, NRL and PDR have been considered and is observed that the AODV-ES performs better as compared to AODV.

\section{PERFORMANCE EVALUATION PARAMETERS}

Packet Delivery Ratio (PDR), End-to-End delay and Normalized Routing Load (NRL) have been considered to compare the performance of AODV-ES, an effective and scalable routing protocol with basic AODV. We explain the following performance matrices to the effect of scheduling algorithm.

$>$ Packet Delivery Ratio (PDR): It defines as a percentage of data packets delivered at receiver end to that of no. of data packets sent for that node. PDR is used to measure the reliability, effectiveness and efficiency of routing protocols

$>$ End-to-End delay: This is the average delay for a packet to traverse from a source end to a destination end. It is measured as the time elapsed from the time when a data packet is originated from a source and it is successfully received by receiver.

$>$ Normalized Routing Load (NRL): It is the number of route control packets per data packet delivered at destination end. It is important to measure the scalability of routing protocol; the adaption to low bandwidth environment and its efficiency in relation to sensor node battery power. Sending more routing packets may increase the probability of packet collision. As a result end-to-end delay may increase and decrease the PDR as well.

In this paper through exhaustive simulations, we measured all these performance evaluation parameters carried out with different mobility rates and number of nodes in the wireless ad hoc network.

The higher PDR and lower end-to-end delay ensure better performance of the AODV-ES. In this paper, we simulated ondemand protocol viz. AODV and AODV-ES on different combinations of mobility rates. It evaluates the performance improvement achieved for AODV-ES in comparison to AODV.

\section{SIMULATION PARAMETERS}

The NS-2 simulation environment [14] offers great flexibility in investigating the characteristics of sensor networks. By leveraging the existing mobile networking infrastructure, we added the capability to simulate sensor networks. [15]One fundamental aspect of sensor networks added in NS-2 is the notion of a phenomenon. The application defines how a sensor will react once it detects its target phenomenon. As long as it continues to detect the phenomenon, a sensor node may periodically send a report to some data collection point. With NS2 , it has been provided the facility to invoke sensor applications by phenomena. We modeled the presence of phenomena in NS-2 with broadcast packets transmitted through a designated channel. In the NS-2 environment, a sensor network can be built with many of the same sets of protocols and characteristics as those available in the real world. The wireless model also includes support for node movements and energy constraints. So we considered NS-2 simulator to simulate performance evaluation and comparison using under Linux operating system. Simulation parameters with their values are described in the Table-1. The NS2 instructions are used to define the topology of the network, 
movement of nodes and to configure source and receiver. The source-destination pairs are spread randomly over the network.

\section{Table -1: Simulation Parameters}

\begin{tabular}{|l|l|l|}
\hline S. No. & Parameters & Values \\
\hline 1 & Area size & $500 \times 500 \mathrm{~m}$ \\
\hline 2 & Transmission range & $250 \mathrm{~m}$ \\
\hline 3 & No. of Nodes & 100 \\
\hline 4 & Mobility Rate & $1,5,10,15,20 \mathrm{~m} / \mathrm{s}$ \\
\hline 5 & Pause times & $10 \mathrm{~s}$ \\
\hline 6 & Data rate & $1 \mathrm{Kbps}$ \\
\hline 7 & Simulation Time & $600 \mathrm{sec}$. \\
\hline 8 & No. of experiments & 5 times. \\
\hline
\end{tabular}

\section{RESULTS AND DISCUSSIONS}

It may consume less overhead of routing protocol when it gets the route to destination information before reaching at the end and generally third party route reply follows this principle to get the information about the route. Further the overhead may be controlled by using TTL-based local recovery. So the NRL may less as compared to basic AODV in WASN. As a result, the performance of AODV-ES is better than AODV, which is noticed from the figure 1 .

Using the third party reply model the intermediate node sends the route reply, if it has route to destination. So the end-toend time delay during route discovery is minimized. As per figure-2, the end-to-end delay of AODV-ES is better in all respect of mobility rates. In case of various mobility rates AODV-ES in WASN performs better than AODV in respect to end-to-end delay.

Now, we observed the PDR of AODV-ES and conventional AODV in various mobility rates in WASN. It is noticed from the figure3 that, PDR of AODV-ES achieves a better result as compared to AODV in respect to various mobility rates. As regards to PDR, AODV-ES proves to be better than AODV for all combinations of mobility rates. It may happen due to less overhead and time delay by using Third Party Reply and TTLbased local recovery. Hence the proposed protocol of AODV-ES outperforms AODV.

From the above discussion we observed that our proposed work AODV-ES routing protocol is better than AODV for Wireless Ad hoc Sensor Network.

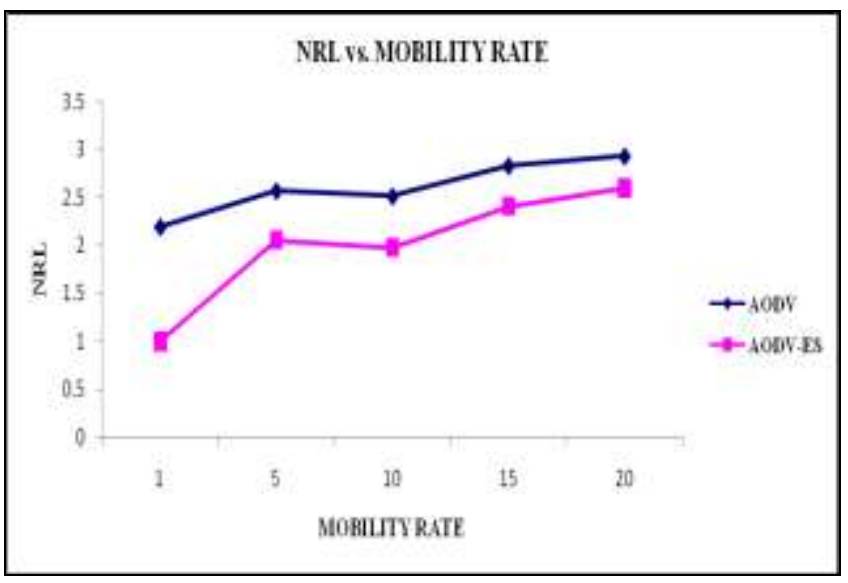

Figure1: NRL vs. Mobility Rates

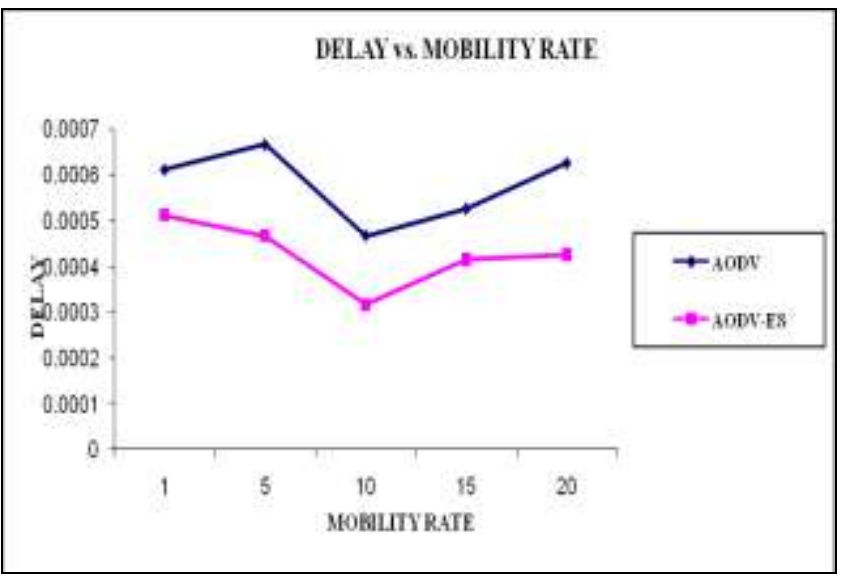

Figure2: Delay vs. Mobility Rates

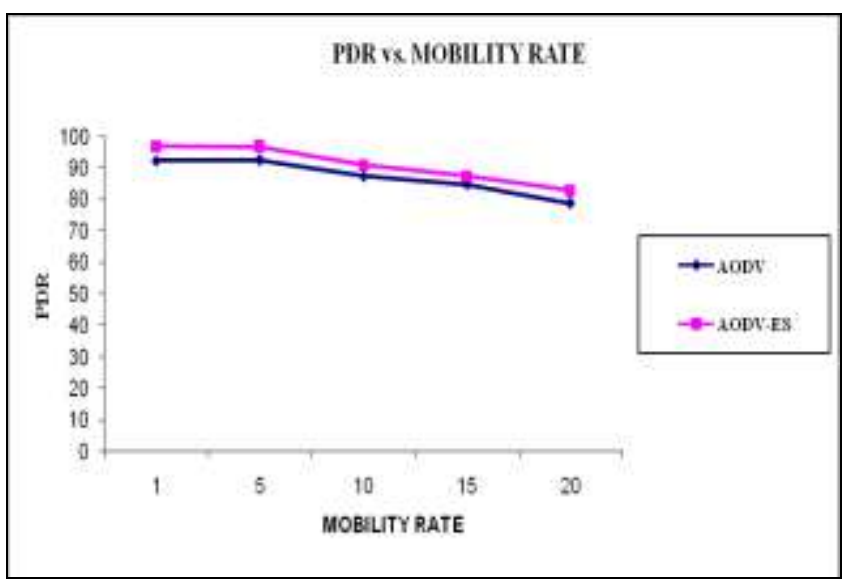

Figure3: PDR vs. Mobility Rates 


\section{CONCLUSION}

The goal of this paper is to characterize the performance of proposed protocol based on WASN. We tried to control the overhead and minimize the time delay with improve effectiveness of AODV-ES. It scales up the effectiveness and efficiency of proposed routing protocol. An Exhaustive simulation experiment reveals that, the proposed AODV-ES protocol performs better than AODV in terms of PDR, end-to-end delay and NRL for all mobility rates. Therefore, it is concluded that, AODV-ES protocol is suitable for Wireless Ad hoc Sensor Network, where effectiveness with scalability and time sensitive is an important issue.

\section{REFERENCES}

[1] D.B. Johnson and D.A. Maltz, "Dynamic Source Routing In Ad Hoc Wireless Networks," Mobile Computing, Vol. 353. Kluwer Academic, 1996.

[2] D.B. Johnson, D.A. Maltz, and Y.-C. Hu, "The Dynamic Source Routing Protocol For Mobile Ad Hoc Networks (DSR)," Internet Draft, Draft-Ietf-Manet-Dsr-09.Txt, Apr. 2003.

http://tools.ietf.org/html/draft-ietf-manet-dsr-09

[3] C.E. Perkins and T.J. Watson, "Highly Dynamic Destination Sequenced Distance Vector Routing (DSDV) For Mobile Computers", Proceeding Of ACM SIGCOMM Conference On Communications Architectures,London, 1994, Pp:234244,

[4] Perkin, C.E and E.M Royer, "Ad Hoc On Demand Distance Vector Routing”, Proceeding $2^{\text {nd }}$ IEEE Workshop, Mobile Computing, Sys. Apps. 1999, Pp:90-100.

[5] C.E. Perkins, E.M. Belding-Royer, and I.D. Chakeres, "Ad Hoc On Demand Distance Vector (Aodv) Routing," IETF Internet Draft, Nov. 2003

http://www.ietf.org/internet-drafts/draft-ietf-manet-aodv13.txt

[6] Li Xia, Zhaohui Liu, Yue Chang, Peng Sun, “An Improved AODV Routing Protocol Based on the Congestion Control and Routing Repair Mechanism", International Conferenece and Mobile Computing, Jan-2009 pp:259-262.

[7] Yan Bin, Yang, Hong Bin, Chen, "An improved AODV routing protocol for MANETs", Proceedings of the 5th International Conference on Wireless communications, networking and mobile computing, 2009, pp:2918-2921.

[8] Hao Jutao, Zhao Jingjing, Li Minglu, "Energy level and link state aware AODV route request forwarding mechanism research", WSEAS TRANSACTIONS on COMMUNICATIONS 2009, pp:292-299.

[9] Limin Meng, Et Al, “A Novel Ad Hoc Routing Protocol Based On Mobility Prediction", Information Technology Journal 7 (3), 12th International Conference On Information Technology 2008 Asin Network For Scientific Information, 537-540.

[10] David Gan Chye Ong, “Ant Intelligence Routing Algorithm for Mobile Ad Hoc Networks" , Ph. D thesis of Malaysia University of Science and Technology,2004.

http://library.must.edu.my/v3/Catalog-Databases/MUSTTheses/2004/MIT/PDF/DavidGanChyeOng.pdf

[11] S.J. Lee, E.M Royer. \& C.E Perkins, "Scalability study of the Ad Hoc On-Demand Distance Vector Routing Protocol", ACM Wiley International Journal of Network Management, Volume-13 Issue-2, 97-114, March/April 2003

[12] Ziping Liu and Bidyut Gupta, "A Modified Shared-tree Multicast Routing Protocol in Ad Hoc Network", Journal of Computing and Information Technology (CIT), Vol. 13, No. 3, 2005, pp. 177-193.

[13] Sally Floyd, Van Jacobson, Ching-Gung Liu, Steven McCanne, and Lixia Zhang, "A Reliable Multicast Framework For Light-Weight Sessions And Application Level Framing", IEEE/ ACM Transactions on Networking, December 1997.

[14] Ns-2 Manual, internet draft, 2009.

http://www.isi.edu/nsnam/ns/nsdocumentation.html

[15] Ian Downard. Simulating Sensor Networks in ns-2.

[16] S H Manjula, C N Abhilash, Shaila K, K R Venugopal, L M Patnaik, "Performance Of Aodv Routing Protocol Using Group And Entity Mobility Models In Wireless Sensor Networks" , Proceedings Of The International Multiconference Of Engineers And Computer Scientists 2008 Vol Ii Imecs 2008, 19-21 March, 2008, Hong Kong

[17] Katsuhiro Naito, Kazuo Mori, and Hideo Kobayashi, "Evaluation of Power-aware Routing for Sensor Networks with Forwarder Nodes" www.iiisci.org/journal/CV\$/sci/pdfs/I382OFB.pdf

[18] Hamdy Soliman, Mohammad AlOtaibi, "An Efficient Routing Approach over Mobile Wireless AdHoc Sensor Networks", 6th IEEE Consumer Communications and Networking Conference, CCNC Jan. 2009, pp:1-5. 